\title{
Issues of gas dynamic characteristics modeling: a study on a centrifugal compressor model stage
}

\author{
Aleksey Borovkov ${ }^{1}$, Igor Voinov ${ }^{1}$, Yuri Galerkin ${ }^{1}$, Aleksandr Nikiforov², Maksim Nikitin ${ }^{1}$, Olga Solovyeva ${ }^{1, *}$, and Kirill \\ $\mathrm{Kabalyk}^{3}$
}

${ }^{1}$ Peter the Great St. Petersburg Polytechnic University, Polytechnicheskaya 29, Saint Petersburg, 195251 Russia

${ }^{2}$ Smolensk state agricultural academy, Bolshaja Sovetskaja 10/2, Smolensk, 214000 Russia

${ }^{3}$ Lodz University of Technology, Institute of Turbomachinery Wolczanska 219/223 Lodz, Poland 90-924

\begin{abstract}
The paper presents the results of CFD-calculations of a centrifugal compressor stage with a high-pressure 3D impeller and a vaneless diffuser. The stage was designed by Prof. A. M. Simonov in the Problem Laboratory of Compressor LPI according to the following design parameters: flow rate coefficient 0.080 , loading factor 0.74 , and the relative Mach number 0.78 . Two design grids were used: 2.4 and 4.4 million cells for the sector with one blade. The entire stage was calculated with a sparser grid. Special "Stage" interface conditions are used to interface the gas-dynamic parameters at the boundary regions. The SST turbulence model was used in the calculations. The results of efficiency characteristics and work coefficient comparison showed the following: in design flow rate all three variants of the calculation overstate the loading factor by $14.3 \%$; the calculated characteristics of polytrophic work coefficient in the staging of 360 degrees are closest to the experimental characteristics, but the absolute value is greater than $12 \%$ at a flow rate coefficient of 0.085 ; the maximum calculated efficiency of a stage (the circle of 360 degrees) is almost equal to the measured maximum efficiency.
\end{abstract}

\section{Introduction}

The total power of centrifugal compressors in the industry of the Russian Federation accounts for tens of millions kW (the gas industry of the Russian Federation alone has approximately 55 million $\mathrm{kW}$ ). The annual capacity addition for the development of production and replacement of obsolete machines exceeds one million $\mathrm{kW}$. The unit capacity of industrial centrifugal compressors is measured in thousands of $\mathrm{kW}$ (up to 32 thousand $\mathrm{kW}$ or more for compressors of the gas industry), which is why many compressors are produced according to individual projects. "Gas Dynamics of Turbo Machines" laboratory of the Joint Science and Technology Institute with the participation of the Institute of Advanced Manufacturing Technologies improves its own engineering methods for optimal gasdynamic design and carries out projects assigned by constructors of compressors [1, 2, 3, 4, 5, 6].

The gas-dynamic project must ensure that the compressor will meet two main requirements:

- obtaining the desired final pressure with the necessary weight output;

- a minimum expenditure of energy, i. e. maximum efficiency.
The equations of motion of compressible viscous three-dimensional flow in the flow path are second-order partial differential equations. The modern gas-dynamic project is based on a certain way of summarising the experiments and is conducted in the following order:

- starting stage design: the main dimensions are selected based on a set of rules and one-dimensional thermodynamic calculation with the efficiency which the designer assigns as the expected value based on their experience;

- optimisation, final dimensions: size specification and optimisation by comparing the options with calculation of efficiency according to a particular mathematical model (a set of algebraic equations with empirical coefficients) that significantly simplifies the work process;

- calculation of gas-dynamic characteristics of the compressor for the mode of operation stated in technical specifications, and in the possible range of operating conditions.

\section{Methods}

Modern computational fluid dynamics methods (CFD methods) allow the calculation of gas motion in the flow path. Unlike mathematical models, the calculation results

\footnotetext{
* Corresponding author: solovyeva.oa@yandex.ru
} 
not only in the gas-dynamic characteristics of the compressor, but also in complete information on the flow structure. This allows you to find places with increased pressure losses and to optimise the flow path by changing the shape and size of the flow path. There is only the issue of verification of calculation results to solve.

There are different opinions regarding the reliability of the CFD-calculation of gas-dynamic characteristics of centrifugal compressors and their stages [7 - 20]. The author of the Agile Engineering method [21, 22, 23, 24, $25,26,27,28,29]$ uses these calculations in his method of design. Examples of single-stage transonic and supersonic compressors of gas turbine engines with semi-open 3D impellers are quoted. The authors also have a positive experience of applying CFD-calculations in such compressors [30, 31, 32]. The author of the dissertation reports on successful modelling of the stages of industrial compressors [12]. He used a software package of the GE company. Therefore, it is not possible to check the result and use it.

The authors' own experience and other sources [33, 34, 35, 36, 37, 38, 39, 40, 41, 42] indicate the twofold nature of the results. The motion of gas in the stator elements of the compressor stage is modelled correctly. CFD-calculations have been successfully used in research and design works [36, 37, 38, 39, 40, 43]. At the same time, characteristics of the stage calculated by commercial packages of ANSYS CFX and Numeca Fine/Turbo are entirely different from the experimental ones.

\subsection{The aim of the work}

The aim of this work is to compare the experimental gasdynamic characteristics of the model centrifugal compressor stage with characteristics calculated by the ANSYS CFX package with different methods of calculation. If successful, the opportunity opens for complete rejection of physical experiments when developing model stages and flow paths of multi-stage compressors.

\subsection{Research objects}

The stage was developed and tested by the scientific group of Prof. A. M. Simonov in the Problem Laboratory of Compressor LPI with the aim of developing methods of calculation and design and exploring the possibility of applying stages with high-head semi-open impellers for industrial compressors [2]. Semi-open impellers in industrial compressors are not widely used, but the object is interesting for turbocharging of ICE.

Object of modelling is presented in Figure 1.

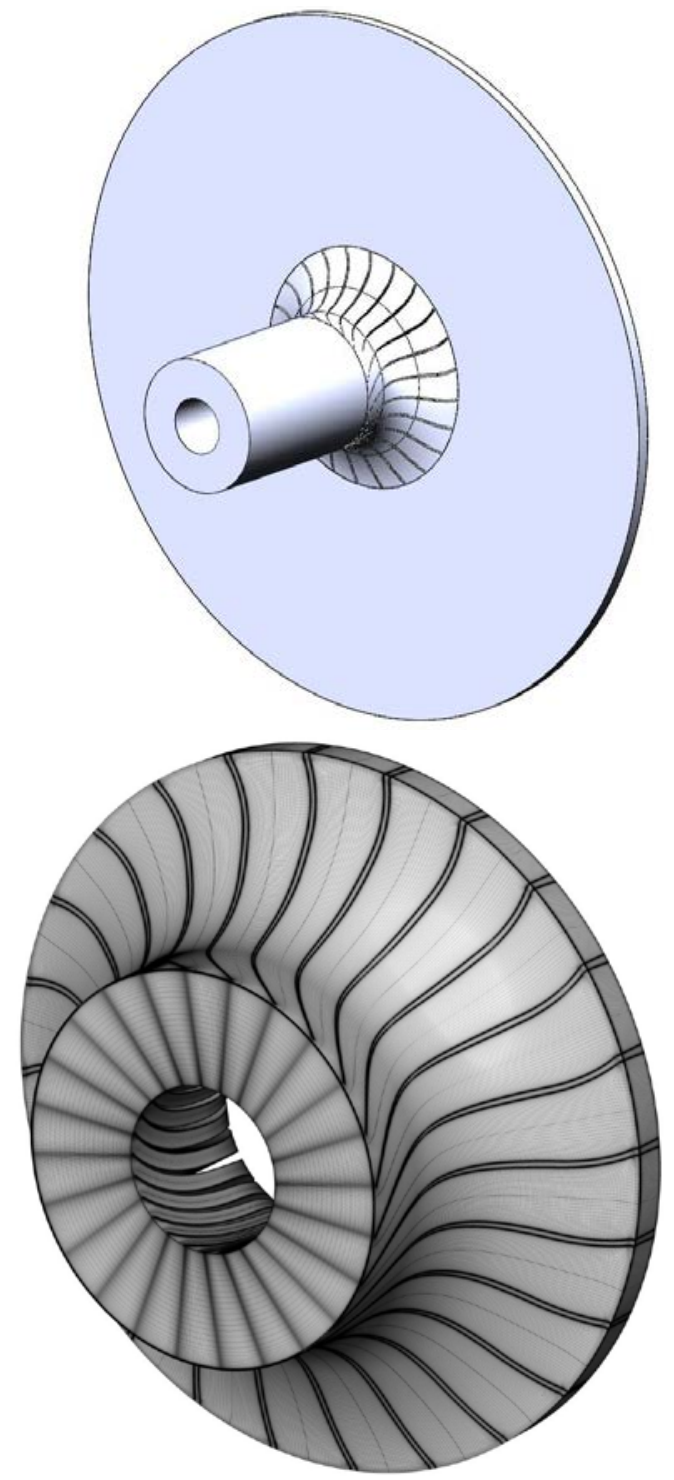

Fig.1. Flow path of the 3D impeller + vaneless diffuser (VLD) and impeller with grid model

The stage with a 3D impeller, a vaneless diffuser, and a scroll is designed for the following calculated parameters: design flow rate coefficient $\Phi_{\text {des }}=0.080$, design loading factor $\psi_{\text {T des }}=0.74$, relative Mach number $M_{u}=0.78$. The 3D impeller has an outlet diameter of $\mathrm{D}_{2}=410 \mathrm{~mm}$. The shape of the 3D impeller is close to the "inducer + radial star" scheme. The blades in the radial part have the form of radial plates with the outlet part bent against the direction of rotation. The outlet angle of the blades $\beta_{\mathrm{bl} 2}=63^{\circ}$, the number of blades is 24.

Characteristics of model stages are presented in a non-dimensional form, for example, as $\eta^{*}, \psi_{\mathrm{i}}, \psi_{\mathrm{p}}^{*}=\mathrm{f}(\Phi)$ [44], where $\eta^{*}$ is total efficiency, $\psi_{i}$ is work coefficient, $\psi_{p}$ is total polytrophic work coefficient. Depending on the size of the compressor and the operating conditions, the transition to dimensional parameters from the non-dimensional characteristics is carried out according to the following equations: 


$$
\begin{gathered}
\bar{m}=0,785 \Phi \cdot D_{2}^{2} \cdot u_{2} \frac{p_{0}^{*}}{R T_{0}^{*}}, \\
p_{0^{\prime}}^{*}=p_{0}^{*}\left[1+(k-1) \psi_{i} M_{u}^{2}\right]^{\frac{k}{k-1} \eta^{*}}, \\
N_{i}=\bar{m} \cdot \psi_{i} \cdot u_{2}^{2},
\end{gathered}
$$

where $\bar{m}$ is mass flow rate, $u$ is blade velocity, $p^{*}$

is total pressure, $T^{*}$ is total temperature, $R$ is gas constant, $k$ is isentropic coefficient, $N_{i}$ is power transmitted to gas by impeller (internal).

When processing the results of the stage test and CFD-calculations, the flow rate is calculated according to the equation (1). Polytrophic total efficiency is calculated by the (4) formula, work coefficient according to the (5) formula, polytrophic work coefficient - according to the (6) formula:

$$
\begin{gathered}
\eta^{*}=\frac{\ln \left(\frac{p_{\text {out }}^{*}}{p_{\text {inl }}^{*}}\right)}{\frac{k}{k-1} \ln \left(\frac{T_{\text {out }}^{*}}{T_{\text {inl }}^{*}}\right)}, \\
\psi_{i}=\frac{c_{p}\left(T_{\text {out }}^{*}-T_{\text {inl }}^{*}\right)}{u_{2}^{2}}, \\
\psi_{p}^{*}=\psi_{i} \cdot \eta^{*},
\end{gathered}
$$

where $c_{p}$ is heat capacity at constant pressure.

\section{Results}

The results of the experiment are presented in Table 1.

Table 1. Measured characteristics of the 3D impeller + VLD

\begin{tabular}{|c|c|c|c|c|c|c|}
\hline & $\mathbf{1}$ & $\mathbf{2}$ & $\mathbf{3}$ & $\mathbf{4}$ & $\mathbf{5}$ & $\mathbf{6}$ \\
\hline$\Phi$ & 0.067 & 0.073 & 0.080 & 0.089 & 0.101 & 0.113 \\
\hline$\psi_{\mathrm{p} 2-2}^{*}$ & 0.746 & 0.730 & 0.713 & 0.690 & 0.640 & 0.430 \\
\hline$\psi_{\mathrm{p} 4-4}^{*}$ & 0.693 & 0.690 & 0.680 & 0.656 & 0.603 & 0.420 \\
\hline$\eta_{2-2}^{*}$ & 0.929 & 0.932 & 0.939 & 0.929 & 0.873 & 0.656 \\
\hline$\eta_{4-4}^{*}$ & 0.866 & 0.870 & 0.877 & 0.870 & 0.823 & 0.573 \\
\hline$\psi_{\mathrm{i}}$ & 0.8037 & 0.783 & 0.760 & 0.743 & 0.733 & 0.655 \\
\hline
\end{tabular}

The 2-2 cross section with the measurement of total pressure is located on the diameter of $1.05 \mathrm{D}_{2}$. The $4-4$ cross section with the measurement of total pressure at the outlet from the stage (the outlet from the VLD in this case) is located on the diameter of $1.6 \mathrm{D}_{2}$.

When constructing a calculation model, a structured scheme with hexagonal elements was used. The concentration needed to meet the turbulence model and wall function requirements were applied to borders of the flow path wall, in order to consider the border layer. The first wall cell is sized from 0.002 to $0.004 \mathrm{~mm}$. The expansion ratio is 1.2-1.5. The total number of computational cells was $1,350,525$ for the sector with one blade - sector $360^{\circ} / 24=15^{0}$. The calculations are performed both for the sector and for the whole computational region. Special "Stage" interface conditions are used to interface the gas-dynamic parameters at the boundary regions. The SST turbulence model was used in the calculations.

In order to investigate the influence of the calculation model dimension on the gas-dynamic characteristics, two grid models for the sector of the model stage were examined. In the first model, the number of cells to describe the flow path was 2,429,380 cells, in the second model it was 4,448,897 cells (modified characteristic). The changes mostly affected the grid of the impeller. In total, the element size was reduced by 1.4 times, and in the wall region the size of the first element decreased from $0.005 \mathrm{~mm}$ to $0.002 \mathrm{~mm}$. The number of elements in the gap was increased from 14 to 34 . Because of this, the average value of $\mathrm{y}^{+}$at the blade surface has changed from 15 to 4.

Thus, three options of calculation were compared with experiments:

- RG-15 - a rare grid with one blade channel, and the sector of the vaneless diffuser of 15 degrees,

- RG-360 - a rare grid with the impeller and entire vaneless diffuser

- DG-15 - a dense grid with one blade channel, and a sector of the vaneless diffuser of 15 degrees,

The main part of mechanical power is transmitted to gas by impeller blades. The corresponding nondimensional coefficient is called the loading factor. Its value is determined by the change of the tangential component of velocity and appears from the fundamental equation of turbo machines. In the condition of absence of the tangential component of velocity at the inlet to the impeller, normal for industrial compressors:

$$
\psi_{\mathrm{T}}=\mathrm{c}_{\mathrm{u} 2} / \mathrm{u}_{2},
$$

where $c_{u}$ is tangential component of velocity.

The friction of the shroud surfaces of the disks and the leakage in the labyrinth seal of the shroud additionally conduct mechanical energy, which is taken into account by empirical coefficients $\beta_{\mathrm{fr}}, \beta_{\text {leak }}[45,46]$. The corresponding non-dimensional coefficient is called the work coefficient:

$$
\psi_{\mathrm{i}}=\psi_{\mathrm{T}}\left(1+\beta_{\mathrm{fr}}+\beta_{\text {leak }}\right),
$$

where $\beta_{\text {leak }}$ is coefficient of leakage in the labyrinth seal, $\beta_{f r}$ is disk friction coefficient.

The linear character of the experimental dependence has been $\psi_{\mathrm{i}}=\mathrm{f}(\Phi)$ observed in the first monographs on centrifugal compressors $[45,46]$ and in the dissertation [2] this is confirmed by the analysis of the characteristics of a large number of industrial compressors. The dissertation [47] shows that this consequence of the linear nature of the dependence of the loading factor on the flow rate at the outlet of the impeller $\psi_{\mathrm{T}}=\mathrm{f}\left(\varphi_{2}\right)$. The coefficients $\beta_{\mathrm{fr}}, \beta_{\text {leak }}$, for most industrial 
compressors, are small and do not deviate the dependence $\psi_{\mathrm{i}}=\mathrm{f}\left(\varphi_{2}\right)$ from the linear character much.

The researched stage with a semi-open impeller has no shroud or its seal, so $\beta_{\text {leak }}=0$. The flow in the gap between the main disk and the stator has not been modelled, so $\beta_{\mathrm{fr}}=0$. Thus, the characteristics are calculated for the loading factor, not for the work coefficient.

Figure 2 shows the loading factors based on the calculation results with two types of grid, with the calculation of one blade channel (sector $15^{\circ}$ ) and the blade system as a whole $\left(360^{\circ}\right)$.

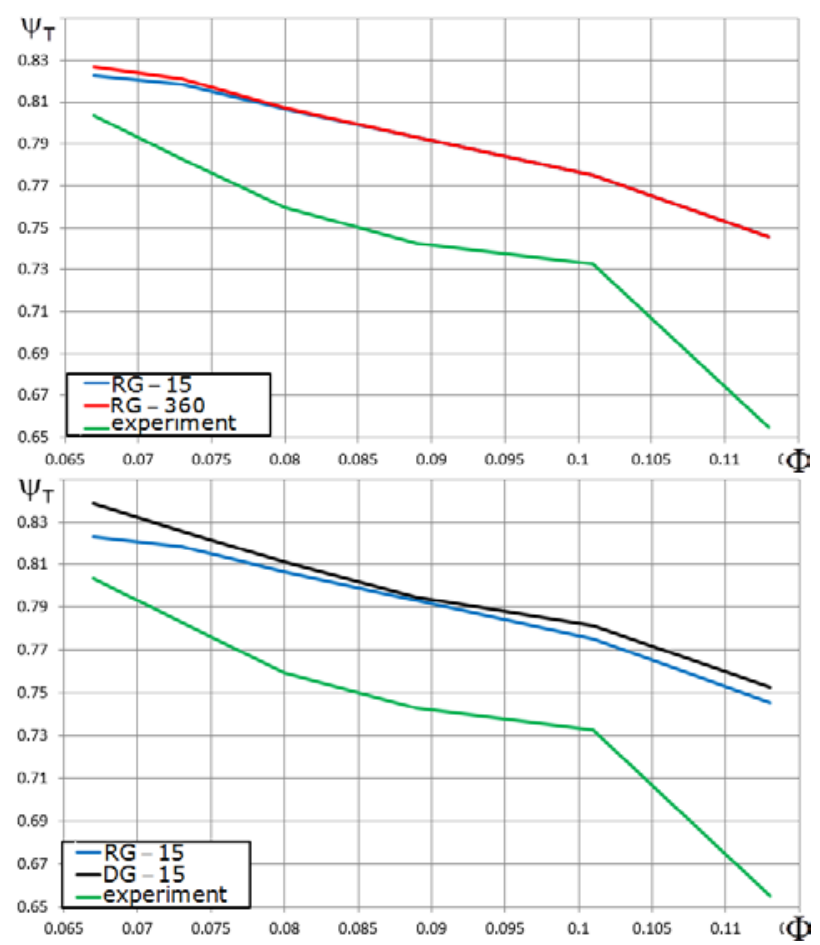

Fig.2. Measured and calculated loading factor characteristics of semi-open 3D impeller

The known results of the calculations in all cases showed an excess of the calculated head coefficient over the measured head coefficient [33, 34, 48]. The angle between the calculated linear characteristic and the $\mathrm{X}$ axis is always bigger or equal to the angle of the experimental characteristic. As with other researchers, our head characteristic lies higher and tilted at a large angle. The characteristic calculated with a denser grid for the $15^{0}$ sector is closer to linear nature.

Figure 3 shows the fields of the absolute speed in the impeller with three flow rates.
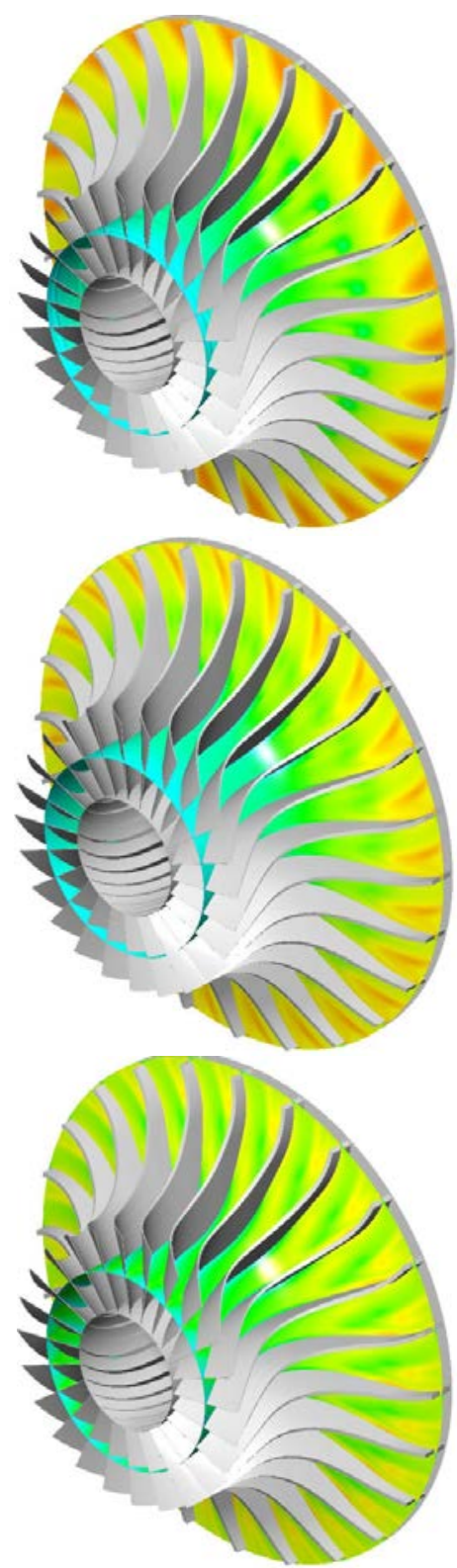

Fig.3. Fields of absolute speed in 3D impeller. On the left $\Phi=$ 0.067 , in the middle $\Phi=\Phi_{\text {pac }}=0.080$, on the right $\Phi=0.113$

Zones of higher absolute velocity at the rear side of the blades are zones of a "trace" [44] a low-energy zone where the relative velocity (velocity in a rotating coordinate system) is small, so the absolute velocity is close to the blade velocity. With the flow rate smaller than calculated and a positive incidence angle, the trace zone is the largest as follows from the experiments and the theory of workflow. Note that in comparison with typical industrial compressor stages, where the outlet angles of the blades are usually much smaller, the work zone of the stage is narrow. The measured maximal polytrophic work coefficient is achieved at $\Phi=0.067$. This corresponds to the boundary of stability - the zone of surge. A larger region of the trace in the calculation experiment also demonstrates the proximity of the boundary of stability

Figure 4 shows the characteristics of the polytrophic work coefficient of the "3D impeller + vaneless diffuser" stage. 


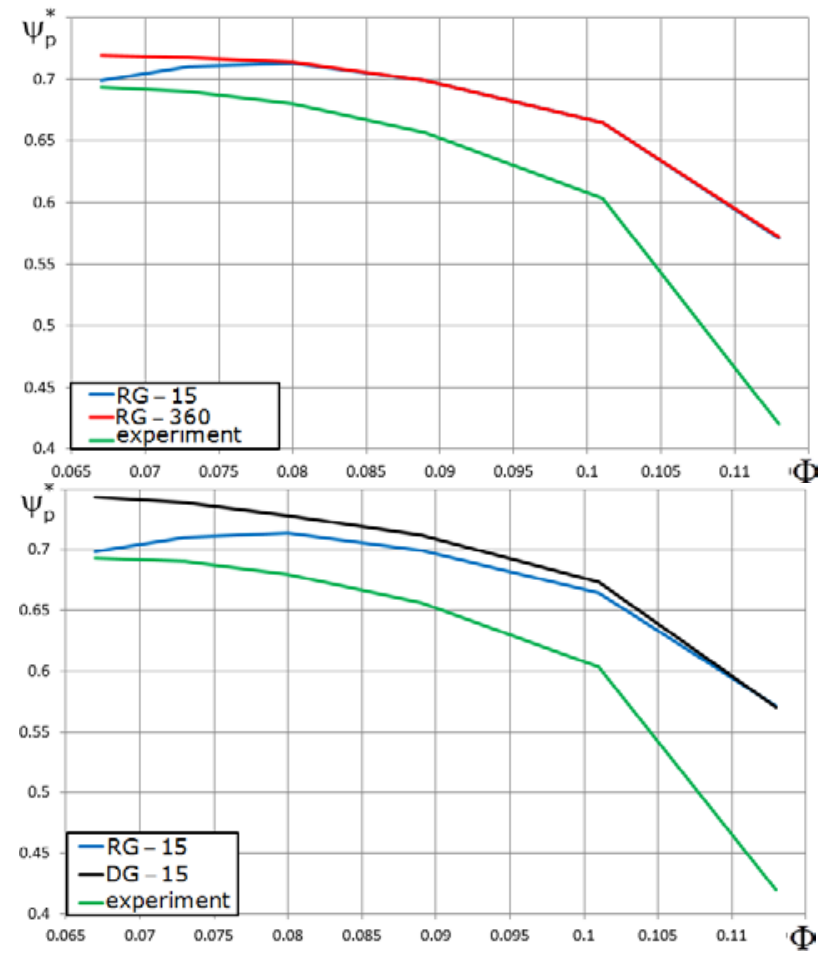

Fig.4. Polytrophic head characteristics of the 3D impeller + VLD stage

The calculation of the $15^{0}$ sector with a "rare" grid yields unsatisfactory results. The maximum head work coefficient with the flow rate $\Phi=0.080$ (this is the surge boundary) clearly does not correspond to the experimental data. The calculation with twice the number of cells in its nature is closer to the experiment, but the polytrophic work coefficient is much bigger, - by about $15 \%$ in design conditions. The closest to the experiment, as for the dependence on the flow and the size, is the calculation of the entire blade system with a "rare" grid.

The calculated characteristics of efficiency in Figure 5 have an expected form, but show a tendency to shift towards a higher flow, as also shown by other researchers.
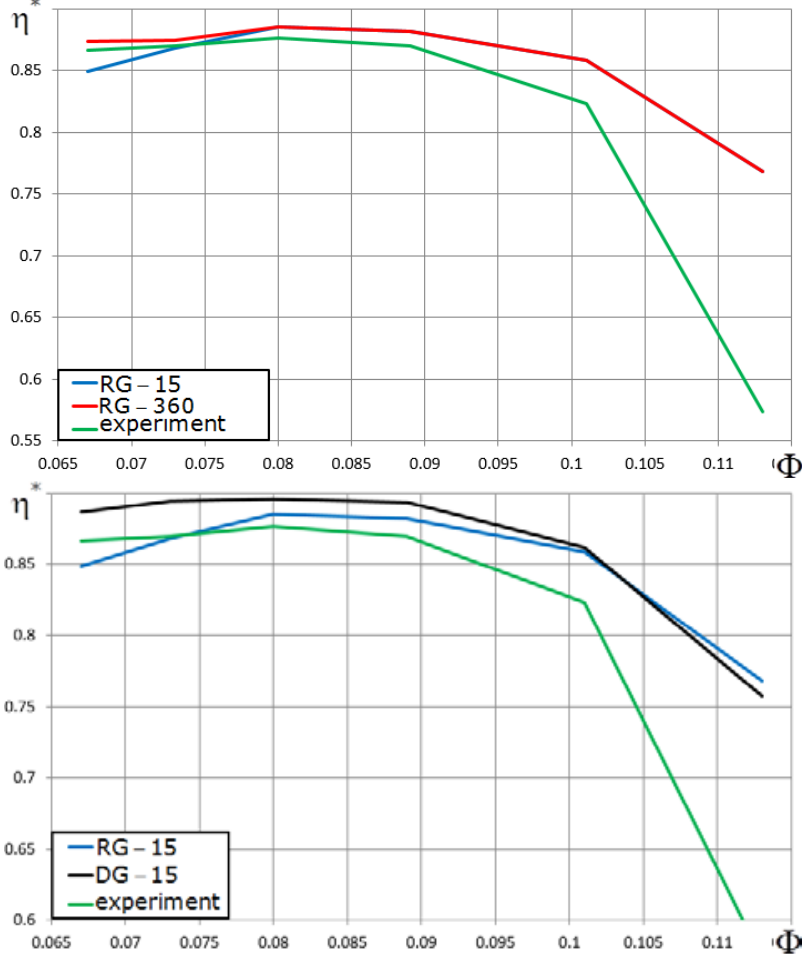

Fig.5. Measured and calculated efficiency characteristics of the 3D impeller + VLD stage

The results of the calculation show the following:

- the RG-15 option showed the worst match, in terms of efficiency, loading factors, and polytrophic work coefficients;

- the RG-360 option showed a satisfactory match of the efficiency and the polytrophic work coefficient and a bad match of the loading factor;

- the DG-15 option showed a satisfactory match of the loading factor, and a bad match of the efficiency and polytrophic work coefficient.

\section{Discussion}

- Option 3 has a characteristic of the loading factor closer to the experimentally confirmed linear character. Options 1 and 2 give the same result - a non-linear characteristic with a deviation in the direction of smaller head coefficient with $\Phi<\Phi_{\text {des }}$. In the design conditions, all three versions of the calculation overstate the loading factor by $14.3 \%$;

- in Option 1 with $\Phi<\Phi_{\text {des }}$, the efficiency decreases rapidly. Therefore, with $\Phi \approx \Phi_{\text {des }}$, the polytrophic work coefficient reaches its maximum. That means that the characteristic calculated under Option 1 has a coincidence of the surge boundary with the design conditions. Such inaccuracy of calculation of the characteristic is not acceptable;

- the form of the polytrophic work characteristic calculated under Option 2 is closest to the form of the experimental characteristic, but the 
absolute value is higher by $12 \%$ with $\Phi_{\text {des }}=$ of 0.085 ;

- the maximum efficiency of the stage calculated under Option 2 is almost equal to the measured maximum efficiency.

\section{Conclusions}

The performed calculation study has confirmed the results previously obtained by the authors when modelling high-head centrifugal stages of gas turbine engines and the results of other researchers. The correct modelling of characteristics of centrifugal compressor stages still remains an unsolved problem. The worst results were obtained by the authors with the most economical calculation, a rare grid, a $15^{0}$ sector. A more expensive calculation with a rare grid and full blade system $360^{\circ}$ evaluates the maximum efficiency level with sufficient accuracy. The authors suggest assessing the capabilities of the most expensive calculation with a dense grid and a full blade system $360^{\circ}$ in the future.

\section{Acknowledgements}

The research was performed by a grant of Russian Science Foundation (project No. 18-79-10165). Calculations involved the supercomputer center "Polytechnical" of SPbPU.

\section{References}

1. Yu.B. Galerkin, Turbocompressors, 596 (2010)

2. K. Soldatova, Dr. of tech. Sci. thesis 257 (2017)

3. Y. Galerkin and K.Soldatova, Gas-dynamic process in industrial centrifugal compressors modeling. Scientific basis, stapes of development, current state, 327 (2011)

4. A. Drozdov and A. Rekstin International Scientific Conference on Energy, Environmental and Construction Engineering (2018)

5. A.A. Drozdov, V.I. Yaroshenko and Y.N. Pisarev, Oil and Gas Engineering, 2141, (2019)

6. .A. Rekstin, Y. Popova and A Ucehovscy Oil and Gas Engineering, (2018)

7. A. Startsev, Yu. Fokin and Eu. Steshakov 29th congress of the international council of the aeronautical sciences (2014)

8. C. Xu and W. J. Chen, Int. Conf. On Jets, Wakes and Separated Flow (2005)

9. J. Kosprdova and J. Oldřich, 20th International Conference on Hydraulics and Pneumatics (2008)

10. R. Puzyrewski, Y.B. Galerkin and P. Flaszynski, $X L$ Internationale Tagung «Forschung Praxis und Didaktik Im Modernen Maschinenbau», pp. 41-48 (2001)

11. R. Matas, EPJ Web of Conferences 180, 02060 (2018)
12. E. Guidotti, $\mathrm{PhD}$ thesis, 100, 2014

13. P. Le Sausse, EPJ Web of Conferences, 45 (2013)

14. R. Matas, T. Syka and O. Lunacek, EPJ Web of Conferences, 143 (2016)

15. P. Harley, S. Spence, D. Filsinger, M. Dietrich and J. Early, Journ. of Turb. 137 (2015)

16. P. Harley, S. Spence, D. Filsinger, M. Dietrich and J. Early, Proc. of ASME Turbo Expo 2015, 65-71 (2015)

17. M. Elfert, A. Weber, D. Wittrock, A. Peters, C. Voss and E. Nicke, Proc. of ASME Turbo Expo 2016: Turbomachinery Technical Conference and Exposition, GT2016-56546 (2016)

18. Z. Xinqian and Z. Meijie, Applied Thermal Engineering 131, 933-946 (2018)

19. I. Shahin, M. Gadala, M. Alqaradawi and O. Badr Proceedings of ASME Turbo Expo 2014: Turbine Technical Conference and Exposition, GT201427336 (2014)

20. U.K. Meduri, K. Selvam and G. Nawrocki, Proceedings of ASME Turbo Expo 2015: Turbine Technical Conference and Exposition, 15-24 (2015)

21. D. Japikse, JANNAF Conference, 331-345 (1996)

22. D. Japikse, 40th Israel Annual Conference on Aerospace Science (2000)

23. D.Japikse and J. Bitter, 11th International symposium on transport phenomena and dynamics of rotating machinery, 511-520 (2006)

24. D. Japikse, JANNAF Conference, 263-275 (1998)

25. D. Japikse and O. Dubitsky, ASME Turbo Expo Conference, 823-834 (2006)

26. D. Japikse, IMechE Conference, 31-50(1986)

27. D. Japikse, K.N. Oliphant and R. Pelton, 10th International symposium on transport phenomena and dynamics of rotating machinery (2004)

28. D. Japikse and M.J. Platt, 10th International Symposium on Transport Phenomena and Dynamics of Rotating Machinery, 132-145 (2004)

29. D. Japikse, JSME fluid engineering conference, (2003)

30. A.V. Grigoriev, A.V. Solovieva, Yu. B. Galerkin and A. F. Rekstin, Compr. Techn.and Pneum. 6, 2127 (2014)

31. A.F. Rekstin, K.V. Soldatova and A.A. Drozdov, Klimovskie readings. Promising areas of the Aeroengine industry, 45-55 (2013)

32. Y. Galerkin, A. Rekstin and K. Soldatova International Conference on Numerical Methods in Industrial Processes. World Academy of science, engineering and technology (2015)

33. D.M. Gamburger, PhD thesis, 190 (2009)

34. A.A. Drozdov, PhD thesis, 236 (2016)

35. Y.Galerkin, I. Voinov and A. Drozdov, Conference Compressors and their Systems (2017) 
36. Yu.B. Galerkin and L.N. Marenina, Compr. Techn.and Pneum., 1, 30-36 (2014)

37. Yu.B. Galerkin and L.N. Marenina, Compr. Techn.and Pneum., 2, 10-15 (2014)

38. Yu.B. Galerkin and O.A. Solovyova, Compr. Techn.and Pneum, 3, 35-41 (2014)

39. Yu.B. Galerkin, O.A. Solovyova, Compr. Techn.and Pneum, 4, 15-21 (2014)

40. L.N. Marenina, Compr. Techn.and Pneum. 3, 27-35 (2016)

41. K. Soldatova International Conference on Compressors and their Systems (2017)
42. A. Borovkov, I. Voynov, A. Kirillov and A. Drozdov, MATEC Web Conf (2018)

43. O.A. Solovyova and Yu.B. Galerkin, Compr. Techn.and Pneum, 3, 10-13 (2017)

44. K.P. Seleznev and Y.B. Galerkin, Centrifugal compressors, 271 (1982)

45. V. F. Ris, Centrifugal compressors, 336 (1964)

46. V.F. Ris, Centrifugal compressor machines, 351 (1981)

47. A.N. Karpov PhD thesis, 137 (2011)

48. K.A. Kabalyk, Master's Degree, 164 (2012) 